\title{
OLOMOUC - POSSIBILITIES OF GEOVISUALIZATION OF THE HISTORICAL CITY
}

\author{
Stanislav POPELKA ${ }^{1}$, Alžběta BRYCHTOVÁ ${ }^{1}$ \\ ${ }^{1}$ Palacký University in Olomouc, Faculty of Science, Department of Geoinformatics \\ Tř́ida Svobody 26, Olomouc, Czech Republic \\ standa.popelka@gmail.com, alzbeta.brychtova@upol.cz
}

Keywords: Geovisualization, Spatio-temporal change, 3D, 3D map, Fortress, Olomouc

\begin{abstract}
Olomouc, nowadays a city with 100,000 inhabitants, has always been considered as one of the most prominent Czech cities. It is a social and economical centre, which history started just about the 11th century. The present appearance of the city has its roots in the 18th century, when the city was almost razed to the ground after the Thirty years' war and a great fire in 1709. After that, the city was rebuilt to a baroque military fortress against Prussia army. At the beginning of the 20th century the majority of the fortress was demolished. Character of the town is dominated by the large number of churches, burgher's houses and other architecturally significant buildings, like a Holy Trinity Column, a UNESCO World Heritage Site. Aim of this project was to state the most suitable methods of visualization of spatial-temporal change in historical build-up area from the tourist's point of view, and to design and evaluate possibilities of spatial data acquisition. There are many methods of 2D and 3D visualization which are suitable for depiction of historical and contemporary situation. In the article four approaches are discussed comparison of historical and recent pictures or photos, overlaying historical maps over the orthophoto, enhanced visualization of historical map in large scale using the third dimension and photorealistic 3D models of the same area in different ages. All mentioned methods were geolocalizated using the Google Earth environment and multimedia features were added to enhance the impression of perception. Possibilities of visualization, which were outlined above, were realized on a case study of the Olomouc city. As a source of historical data were used rapport plans of the bastion fortress from the 17th century. The accuracy of historical maps was confirmed by cartometric methods with use of the MapAnalyst software. Registration of the spatial-temporal changes information has a great potential in urban planning or realization of reconstruction and particularly in the propagation of the region and increasing the knowledge of citizens about the history of Olomouc.
\end{abstract}

\section{INTRODUCTION}

Visualization of historical objects, cities and landscapes are used in many fields of human activity, but especially in archaeology and tourism. However, most of the projects leave aside the possibility of comparison the historical state with the present (e.g. virtual exploration of the city of Livorno [3]. Thus, the user loses connection to the reality and then it is hard to imagine where the objects stood. Comparison of the past with the present is often done through a pair of photographs or paintings depicting the same place in different time periods. In most cases, a printed publications (e.g. [5]) or calendars are the media, where the spatial information is missing so the user knowledge of the particular place is required. Another and more perspective is the variant of the visualization of georeferenced historical maps overlaying the current map or orthophoto. By changing the transparency of the old maps the historical situation can be directly compared with the modern. These maps can be viewed on a large number of mapping portals, or as a layer in Google Earth, so maps are available to the general public. Today, computer-generated perspective views of cartographic content is widespread, often named as 3D maps. The development of geoinformation technologies has facilitated the creation of graphic representations and therefore they are used not only by experts in the field of geoinformatics or cartographers, but also by the general public. As the Haeberling [10] states, perspective perception of a generalized and symbolized geographic space offers often a better understanding of spatial coherences. 3D maps could be seen as a supporting complement of classic orthogonal maps. Except a digital representation of the contemporary state, which can be used e.g. for educational purposes [20,21], it is possible to use 3D maps to represent the historic city's face e.g. [16] or country's [17]. Thanks to the 3D perception, the user can better imagine the spatial context. The physical model, such as very popular bronze models of cities, loses connection to reality, whereas in the case of the digital model it can be viewed in the context of map or the orthophoto. A virtual variant of the 3D model of the town was in the focus of the project Rome Reborn [8] carried out by experts from Italy and the USA. During the project the city of Rome was completely created in 3D as it was in $320 \mathrm{BC}$. The buildings were modeled in height detail, despite of the fact that for 
most buildings there have not been accessible source of information about how these buildings looked like. 3D models can also present how the city would developed if there would not been an engineering boom and the destruction of historic buildings, as in the case of Melbourne [4]. However, more often is a virtual reconstruction of urban areas or individual buildings (e.g. Honselaarsdijck Palace [1], 3D-ARCH project [19]). There are many methods of 2D and 3D visualisation which are suitable for depiction of historical and contemporary situation and for following spatio-temporal changes of built-up area. These methods have various levels of reality approximation. The focus of the project was put only on the capabilities of the virtual (computer-based) environment. This article briefly describes methods of geovisualization of spatial-temporal changes in the city. Geoviosualization is in this case understood as a set of cartographic tools and techniques supporting processing of data with geographical (spatial) or time component (According to ICA). The project outputs have been tested on a case study comparing historical and contemporary face of the city of Olomouc, which has changed over the past 150 years from a strong baroque fortress to the modern city. The motivation of the project is to introduce this fundamental change to the general public, and remind the history of the city of Olomouc.

\section{SPATIAL DATA AND INFORMATION SOURCES}

For the processing with the informational content of visualization methods many sources of information and maps have been used. Scientific literature documenting the historical context and descriptions of the fortress objects was used [15, $23,6]$. Pairs of images were taken from the publication Olomoucke promeny [5]. The most important source of spatial information has been Rapport plan from the year 1842. It is available in Kriegsarchiv in Vienna and for the purposes of this paper was lent by the MOF's Ltd. Rapport plan contains a description of the fortress core (alias noyau), there is only floor projection of individual buildings. In determining the approximate height of the buildings knowledge of the preserved buildings height and drawings in the book "Fortress Olomouc" [15] were used. The last sources of information were historical plans of specific objects. However, they were available for only a few buildings. In the case of 3D digital reconstruction of the historic fortifications of the city of Olomouc the only possible method to use was the method of Empirical Techniques. Most of the walls do not currently exist. Only historic maps and sources for landscape reconstruction were used without doing extensive research on archaeological reality, where high degree of truthfulness is required.

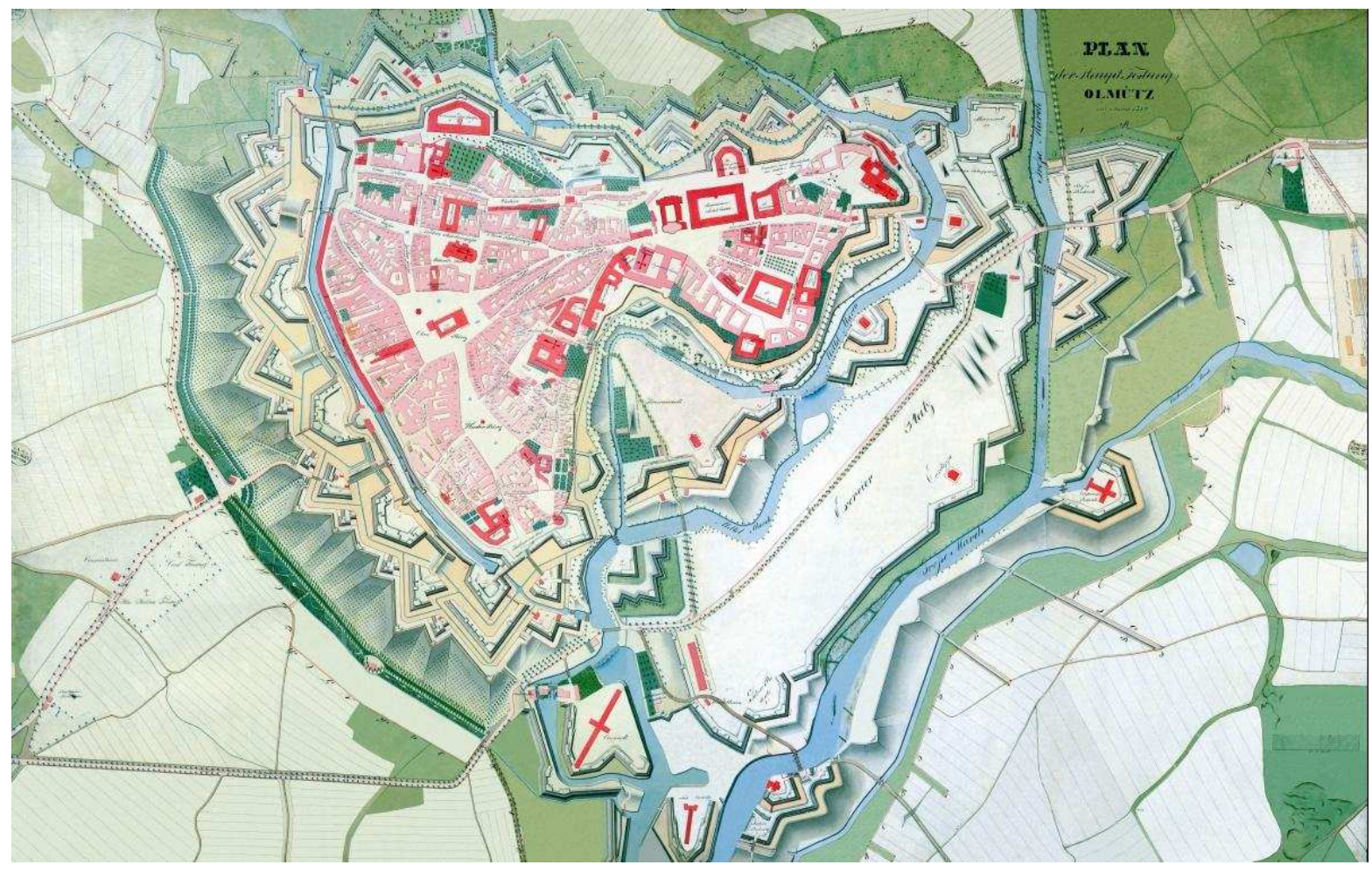

Figure 1: Rapport plan of Olomouc from year 1842. 


\section{GEOVISUALIZATION METHODS}

The aim of this project is to create an interactive application used to visualize the spatial-temporal changes in the historical city of Olomouc, using five visualization methods - textual information, a comparison of historical and contemporary photographs, georeferenced historical maps overlaid over current orthophoto and particularly 3D map of the bastion fortress. The technique of photorealistic 3D model has only been tested on a few objects because of the high time demands and lack of appropriate data. Essential goal is to bring near a period of 150 years ago to ordinary internet users by providing a well known Google Earth environment. The advantage of using Google Earth is a possibility of spatial localization. Not only maps can contain the spatial information but also historical and contemporary photographs or text paragraphs describing the fortress object can be geolocalized.

\subsection{Textual information}

Textual information can provide a very detailed and accurate description of spatial-time changes. Compared to other methods mentioned below, its disadvantage lies in demands on user to apprehend the information from the text and the inflexibility of communication skills in the perspective of different languages. The bases for the preparation of the textual description of spatial-temporal changes were taken from the scientific literature. Presentation of the textual information uses visualization environment of Google Earth and the KML language. The text is presented via the KML Placemark element, which contains the definition of the geographic location and the style of information bubbles appearance, whose presence and navigating to can be interactively controlled by the user. The publication of the text in bubbles follows the rules of HTML and CSS.

\subsection{Pictorial information}

Pictorial, especially photographical documentation is a simple procedure for recording the current state of the architectural object. Before the invention of the photography it is difficult to say with certainty that the image is a faithful replica of the historic state. The paintings and engravings were created as works of art, or biased, and thus often capture only half-truths. Therefore, when reconstruction of the historic state it is necessary to approach the nonphotorealistic works with a caution. The credibility of such information should be supported by archaeological research or period maps. Image content can be divided into two main categories - perceptual content and semantic content [13]. Perceptual content includes attributes such as colour, intensity, shape, texture, and their temporal changes, whereas semantic content means objects, events, and their relations [11]. Both components of visual perception are important to monitor spatial-temporal changes. Comparison of the current and historical status via a pictorial documentation requires the acquisition of pairs of images of the same object. Used pairs of images were taken from the book Olomoucke promeny [5]. In the light of the fact that contemporary photographs were taken in this publication without the need for more accurate comparisons with historical, some couples has been due to entirely different viewing angle completely useless. Some historical pictures had to undergo a slight transformation (the feature Distort in Adobe Photoshop was used) to fit better in the comparison with the modern photos. For the purpose of this visualization method a simple application were developed using Adobe Flash and Action Script. The application allows interactive comparison of two images. The principle is based on user-controlled opacity changing of contemporary image overloaded on the historical figure. Each application are, similar to a text expression methods, presented using the KML Placemark element in the Google Earth environment. The same procedure is used, when CDATA sections, which defines the reference to the Flash SWF file, is inserted into the body of Placemark.

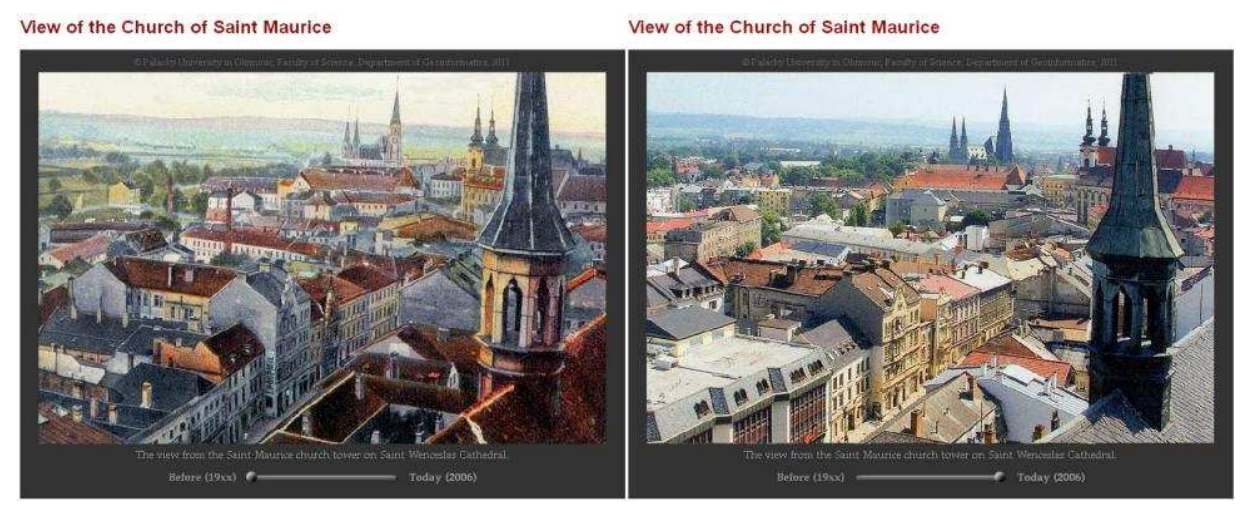

Figure 2: Flash application for comparing two images from different time periods. 


\subsection{Map}

Unlike photographs, maps are abstract models of reality and involve transformations of various kinds [12]. The main task of the map is the presentation of the spatial information. In general, users use map in different ways: for recognition of particular presented objects, for general orientation in the surroundings and for various map measurements [18]. Cartography is the most important way to transmit and share geographic knowledge. Although map reading requires some experiences with a synthesis of spatial information, the transfer of spatial information from maps is much faster than using text or images [14]. A simple but illustrative and comprehensive method to show spatialtemporal changes is to display the georeferenced historical map over the orthophoto or modern map. The only available historical material for this project is Rapport plan from the year 1842, which was georeferenced through ESRI ArcMap software, using buildings and river confluence as control points. To allow access to the wide public was required to publish georeferenced maps on the Internet. As in the previous two methods the Google Earth visualization environment was used. For this reason, it was necessary to create a KMZ file containing the map tiles. Through this process sufficiently fast to download high-quality maps viewing was maintained. For the cutting map into tiles the freely available tool MapTiler has been used. Very convenient is the possibility of changing transparency of historical maps, and thus allow comparison with the current situation. The user can easily identify historical defunct sites in the context of detailed contemporary topographic maps or orthophoto.
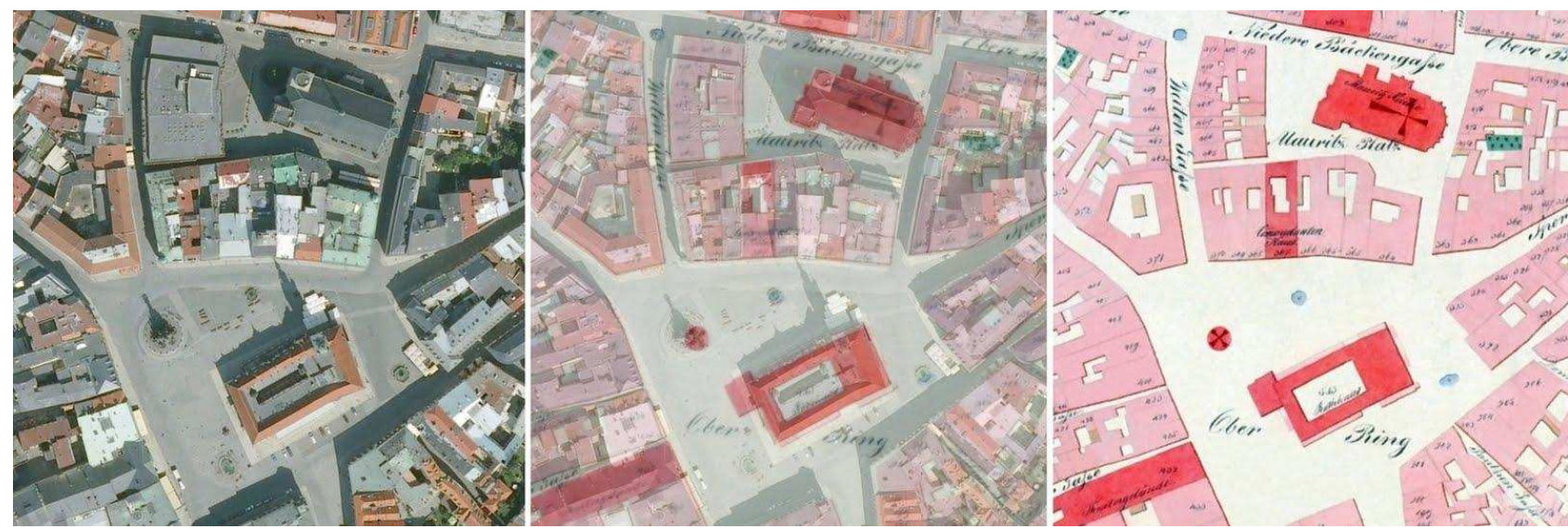

Figure 3: Visualization of the Rapport plan overlaying the orthophoto. The user controlled opacity changing helps to identify historical buildings in the nowadays context.

\subsection{D Map}

Three-dimensional visualizations are well-established for the presentation of maps or landscape models. Today, the need to present three-dimensional (3D) cartographic content on computer monitors is growing and the possibilities for these presentations are increasing (Buchroithner et al. 2011). Because the world is the three-dimensional the perception of $3 \mathrm{D}$ information is more natural and therefore, in some cases, the $3 \mathrm{D}$ visualization is more effective than $2 \mathrm{D}$. In the history it can be traced tendency of using some typical graphic techniques aimed to express the relief of the landscape as realistic as it is possible [18]. Usually it was perspective visualization suggesting 3D effect using shading, crosshatching or hill symbols. 3D map contains both semantic and geometric description of the captured area [24] and its visualization in $3 \mathrm{D}$ environment gives the user a better idea of space, especially height proportions. The approach of spatial-temporal change visualization using 3D map mentioned in this article counts only with the 3D variant of historical maps, when the comparison with the reality is realized only through the possibility of changing transparency of 3D map over orthophoto. In the case of this project, 3D map is considered as a derivation of the above mentioned Rapport plan. Individual fortress objects were modelled in 3D textured using images of historical maps and located exactly at their places on the map. The plastic impression has been reached. Bastions and other buildings pop up out from the map. Users can imagine how the fortress appeared even the 3D map is not a precise representation of the reality.3D map of Olomouc bastion fortress was created in the freely available Google SketchUp. It was created especially for architects, but it is also use as film makers, game developers and many other professions. With the use of Push/Pull tool of Google SketchUp and few other operations almost any shape can be created. These shapes can then be mapped by any texture. In this particular case, as a texture image Rapport plan was used. This is more natural than using photorealistic textures such as grass, bricks, rocks, etc. 

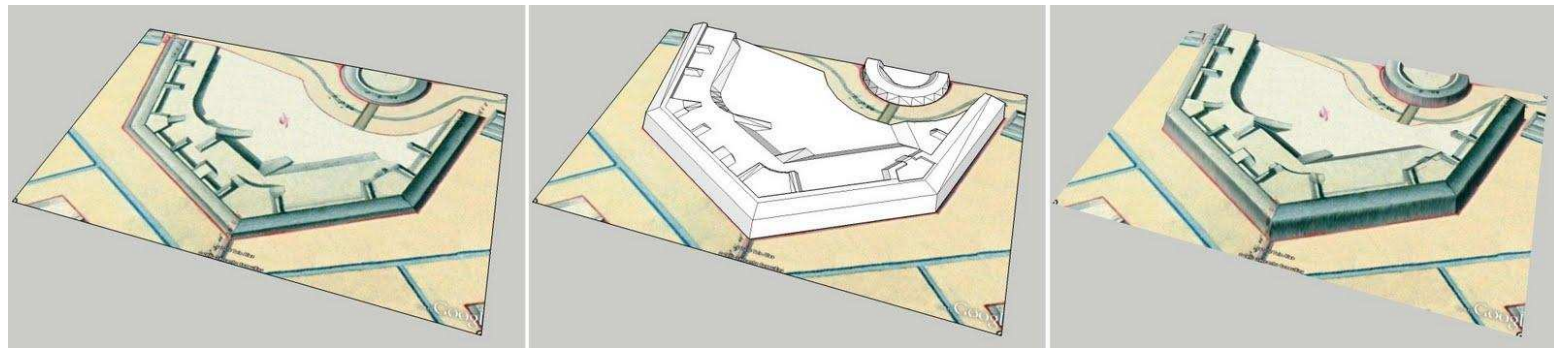

Figure 4: The procedure of 3D map creation on the basis of the Rapport plan.

The creation of the 3D map and modelling shapes of the fortress objects very time consuming activity; currently it is prepared only the southern and south-western part of the fortification.

\subsection{Photorealistic model}

The most perfect and also the most difficult digital representation of the reality is the photorealistic 3D model. While the digital reconstruction of the defunct buildings it is often very difficult to find relevant historical documentation of the exact dimension and shape of the object. The following text briefly describes the modelling techniques that are appropriate to the acquisition of actual photorealistic 3D models. In the case of modelling non-existing objects only empirical techniques can be applied with use of historical plans, photographs or archaeological research results. Large amount of techniques for 3D the digitization of objects exists. The most popular methods are laser scanning and terrestrial photogrammetry. These methods are very expensive and cannot be used for modelling extinct objects. In this case it is necessary to used so-called empirical techniques. The model parameters are then based on historical documents, plans or archaeological research. The creation of detailed worked out textured 3D model of the whole historical city is the work for many people for a long time. For example, the work on above-mentioned Rome Reborn project, which also uses the Google Earth environment, lasted an international team of workers more than ten years [22]. For this reason, just samples of only a few buildings that survived to the present were processed. This is the socalled Teresian gate, Water and the Old town Barracks and the system of walls in Bezruc Park. 3D models were same as the 3D map constructed in an environment of Google SketchUp.

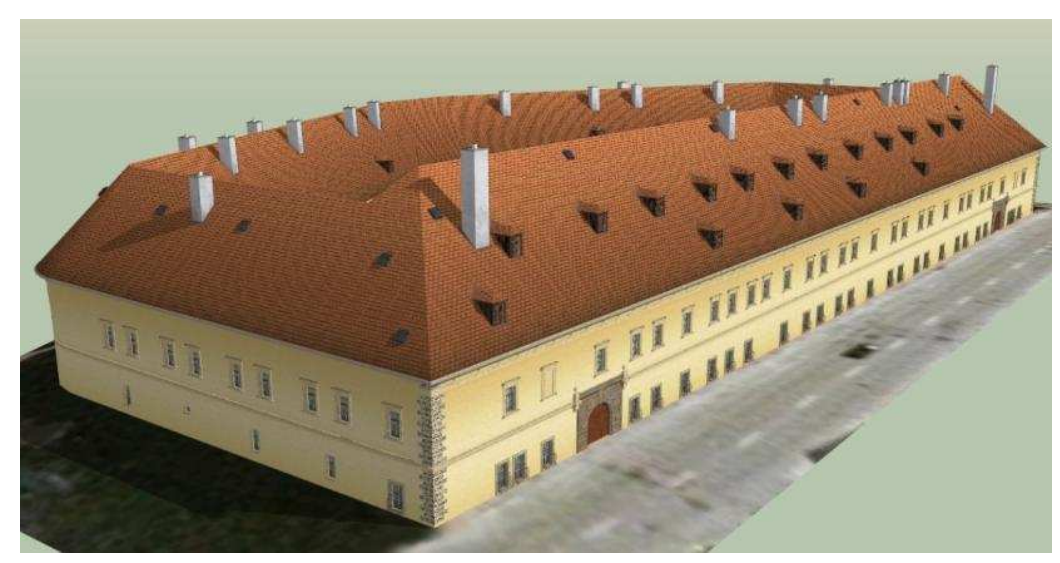

Figure 5: Photorealistic 3D model of Old Town barracks.

\subsection{Interface and application preview}

The Google Earth environment was used for visualization of spatial-temporal changes. Google Earth allows inserting overlays such as masthead, legend, notes, etc... Navigation between layers is realized via a tree-structure in the left pane. Here the user can switch on and off the layer that contains the output from individual visualization methods. For example it is possible to simultaneously view and compare historical map images or text. Using animation several flybys of historic fortifications was created, which are also available. 


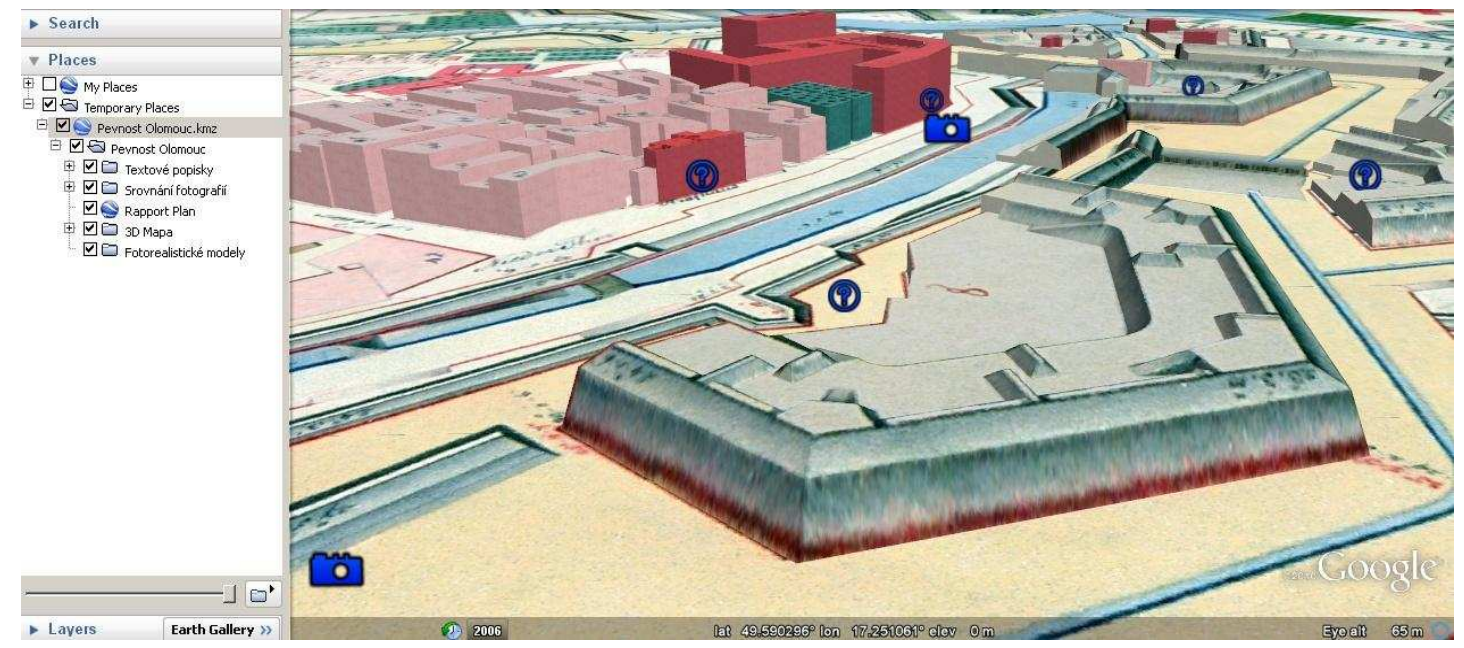

Figure 5: The environment of interactive application in Google Earth.

In interactive and animated map scenes, objects can be modelled and defined by changing graphic or positional attributes. Objects can also easily be created by changing their size or shape. Only with the advent of modern computer technology, map objects can be set in motion easily [9]. This project benefited from the first mentioned option - flight over the terrain with other time-controlled animated actions. Comprehensive guide was created combining all described visualization method, which allows a user to obtain information in a coherent sequence. The base of the interactive guide is to plan the tour route visualization of the informational content in a pre-specified time sequence. The user controls the speed of the tour and the time within which the multimedia blocks of text or image are displayed. An additional multimedia element of the animated flyby can be music or sounds. Examples of such application can be an Interactive Reconstruction of the Roman villa at the Weilberg [7]. This project benefited from the first mentioned option - flight over the terrain with other time-controlled animated actions. Comprehensive guide was created combining all described visualization method, which allows a user to obtain information in a coherent sequence.

\section{EVALUATION OF METHODS}

The evaluation of the five previously mentioned methods of spatial-temporal changes visualization (e. g. text, images, maps, 3D map and photo-realistic 3D model) was done through an expert analysis. The evaluation was divided into two levels. Firstly the technological demanding-ness of the processing and preparation methods was investigated. In the second level the user perception was evaluated. In both levels all factors were assessed separately by their severity and the outcome is determined by the weighted average. Due to necessity of comparison of two evaluation categories, the absolute values of weighted average were converted to a relative, where $100 \%$ corresponds to the highest values. In both categories the highest average was detected in the case of photorealistic 3D model.

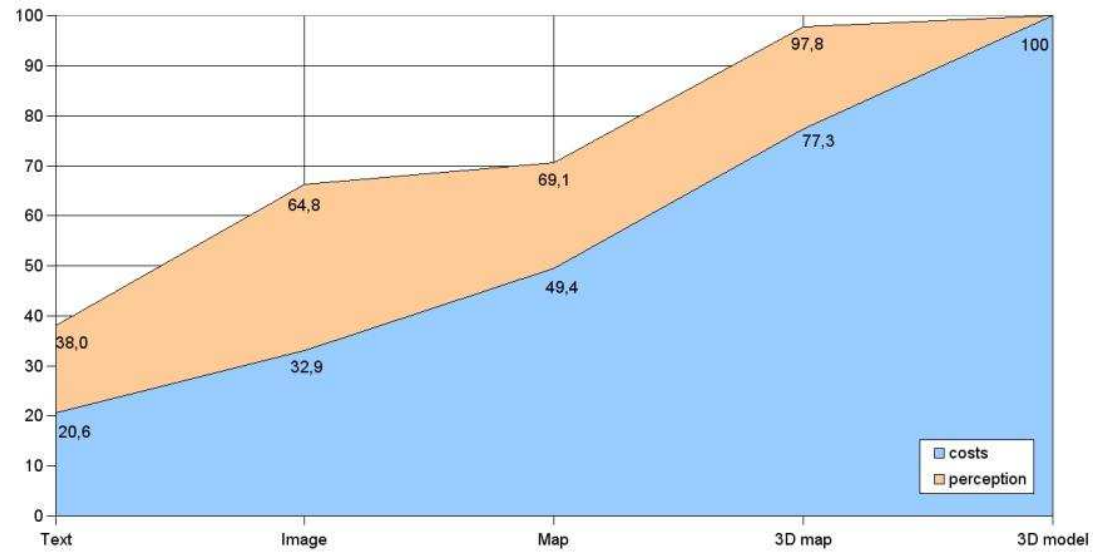

Figure 6: Comparison of technological processing costs and user perception quality. 
Visualization methods evaluation according to the technological processing can be considered as totally objective - it is based on practical experience with creating visualization applications. The second part, where the perception of visualization was evaluated, is based on qualified presumption. The ideal amount of information is expressed by the point where the difference between the information benefit ratio and the cost of information acquisition is maximal, so the best method of spatial-temporal visualization of change would be a pair of images - the difference between the relative costs of processing and effectiveness of the perception in comparison with other described methods is the highest. Anyway, from the user perspective it is much more efficient to use methods with higher visualization potential, despite increasing costs. From this point of view, the best method seems to be 3D maps. Two-dimensional map is inconvenient because of the low increase of the perception quality considering the high cost growth. The same situation can be observed in the case of the photorealistic 3D model.

\section{FUTURE PLANS}

Processing with the visualization of the historic fortification is not yet completed and is still ongoing. As for simple visualization through the text, it is necessary to find the optimal number of labels (informational bubbles) so that the map is not too cluttered. A similar situation arises in the case of images comparison, where the limiting factor is the number of available and suitable photographs or images from the past. In the case of overlaying historical maps, the limiting factor is the difficult situation with the copyright of historical material. The vast majority of Olomouc fortress data and information is owned by the Viennese Kriegsarchiv. Our goal is to obtain the maximum number of plans and maps of Olomouc fortress from different historical periods. The absence of available data source influences creation of 3D maps and 3D models. In this case, the limiting factor is rather the time. 3D modelling is very time consuming and technically demanding work. Fortress consists of a large number of more or less coherent structures. To create 3D maps and 3D models of complete Olomouc fortification it is necessary to create a digital terrain model for the whole Olomouc because of inaccuracies in the underlying digital terrain model contained in Google Earth. Evaluation of visualization techniques, described in the Chapter Spatial-temporal changes visualization methods evaluation, is currently quiet subjective. This should be solved during further research of both authors, which will assess the effectiveness of various types of visualization using the device for eyes tracking. Currently the created application of spatial-temporal visualization of Olomouc city changes is not accessible to the public for reasons of unsolved copyright of historical documents. Future use of application can be seen mainly in the tourism, and in the promotion of the city of Olomouc. The integration into the emerging Museum of the Olomouc Fortress and to the Museum of Science under the auspice of Palacký University is considered. The absolute goal is to create a complete model of the fort and its implementation in an immersive virtual reality system in order to be able to walk through the historic city.

\section{CONCLUSION}

The article describes five methods to express the spatial-temporal change in the historic town and also describes their advantages and disadvantages using the case study of the historic city of Olomouc. 150 years ago the city was a strong fortress, but nowadays a few of the citizens are able to get an idea of the extent of the fortress buildings. All visualization methods use georeferenced data, so the results can be locate in the context of geographical coordinates, which has positive impact on user idea of the historical status of the city.

\section{REFERENCES}

[1] Boer, A., Processing old maps and drawings to create virtual historic landscapes, e-Perimetron, 2010, Vol. 5, No. 2: $49-57$.

[2] Buchroithner et al., Lenticular Creation of Thematic Multi-Layer-Models, Proceedings of GeoViz Workshop, 2011, Hamburg, Germany.

[3] Carrozino, M. et al., The Immersive Time-machine: A virtual exploration of the history of Livorno, Proceedings of 3D-Arch Conference, 2009, Trento, Italy.

[4] Cartwright, W.E., Using 3D models for visualizing “The city as it might be”, Proceedings of ISPRS Technical Commission II Symposium, 2006, Vienna, Austria.

[5] Fiala J. (2006) Olomoucke promeny 2006, Olomouc, Danal, 2006.

[6] Fischer, R., Olomoucka pevnost a jeji zruseni, Olomouc, 1935.

[7] Google Earth Blog, Animated 3D models in Google Earth. In digital form

http://www.gearthblog.com/blog/archives/2010/11/animated_3d_models_in_google_earth.html, 2010

[8] Guidi, G., Frischer, B., Lucenti I., Rome Reborn - Virtualizing the ancient imperial Rome, Proceedings of the 3D Arch Conference, 2007, Trento, Italy.

[9] Haeberling, Ch., 3D Map Presentation - A Systematic Evaluation of Important Graphic Aspects, Proceedings of ICA Mountain Cartography Workshop "Mount Hood", 2002, Mt. Hood, Oregon, USA. 
[10] Haeberling, Ch., Cartographic design principles for 3D maps - A contribution to cartographic theory, Proceedings of ICA Congress Mapping Approaches into a Changing World, 2005, A Coruna, Spain.

[11] Jung K., Kim K.I., Jain A.K., Text information extraction in images and video: a survey. Pattern Recognition, 2004, Volume 37, Issue 5: 977-997.

[12] Keates, J. S., Understanding Maps, London, Longman, 1982.

[13] Kim, H.K., Efficient automatic text location method and content-based indexing and structuring of video database. J. Visual Commun. Image Representation, 1996, 74: 336-344.

[14] Kubicek P., Kozel J., Cartography Techniques for Adaptive Emergency Mapping. Proceedings of Risk Models and Applications Conference, 2010, Berlin, Germany.

[15] Kupka, V., Kuch-Breburda, M., Pevnost Olomouc, Dvur Kralove nad Labem , FORTprint, 2003.

[16] Langweiluv model Prahy, In digital form: http://www.langweil.cz/.

[17] Niederoest, J., A bird's eye view on Switzerland in the 18th Century: $3 D$ recording and analysins of a historical relief model, Proceedings of the CIPA conference, 2005, Antalaya, Turkey.

[18] Petrovic, D., Masera P., Analysis of user's response on 3D cartographic presentations, Proceedings of 5th ICA Mountain Cartography Workshop, 2006, Bohinj, Slovenia.

[19] Remondino, F. et al. 3D virtual reconstruction and visualization of complex architectures - The " $3 D-A R C H$ "

Project, Proceedings of the 3D-ARCH conference, 2009, Trento, Italy.

[20] Swiss Federal Office of Topography (2004) Atlas of Switzerland. Interactive and Multimedia CD-ROM. Ed. Swisstopo, Wabern, Switzerland.

[21] Swiss World Atlas interactive (2010) In digital form: http://www.schweizerweltatlas.ch/en.

[22] Wells, S. Rome Reborn in Google Earth, In digital form http://www.romereborn.virginia.edu/rome_reborn_2_documents/papers/Wells2_Frischer_Rome_Reborn.pdf.

[23] Zatloukal R., Olomoucka pevnost ve svetle archeologickych nalezu, Olomouc Fortess, 2004.

[24] Zebedin, L. et al., Towards 3D map generation from digital aerial images. ISPRS Journal of Photogrammetry and Remote Sensing, 2006, Volume 60, Issue 6: 413-427. 